\title{
Top-down oder Bottom-up?
}

\section{Die Frage nach den Erfolgsbedingungen von Umweltpolitik ist eine typisch transdisziplinäre Herausforderung. Formal ist sie ein Teil der Policy-Analyse. Um die Erfolgsbedingungen umweltpolitischen Handelns zu verstehen, gilt es, sich von isolierten disziplinären Top-Down-Ansätzen zu lösen und sich der Fragestellung mit interdisziplinärer Offenheit und empirisch gestüłzten bottom-up-Ansätzen zu nöhern. Der Beitrag geht auf das Wesen und die Möglichkeiten beider Ansütze für die Policy-Analyse ein.}

$\mathrm{D}$

Von Martin Jänicke e Policy-Analyse ist derjenige Teil der Politikwissenschaft, der sich nicht mit dem politischen System als solchem (,polity") und seinen Willensbildungsprozessen (,politics") befaßt, sondern die konkreten Inhalte der Staatstätigkeit (,policy“) zum Gegenstand hat. Es handelt sich vorwiegend um den Steuerungsund Managementaspekt von Politik, mit einer gewissen Nähe zur Verwaltungswissenschaft. Es geht um die staatliche Bearbeitung gesellschaftlicher Probleme, mit einer starken Spezialisierung auf einzelne Ressorts; Policy-Analyse wird auch mit „Politikfeld-Analyse“ übersetzt.

Im Vordergrund der Policy-Analyse bzw. der Evaluationsforschung steht die Effektivität bzw. Effizienz, aber auch die Fairneß (equity), Akzeptanz und Praktikabilität politischer Maßnahmen und Programme (1). Eine spezielle Richtung der Evaluationsforschung befaßt sich mit Erfolgsbedingungen von Umweltpolitik (2). Die Frage nach den Erfolgsbedingungen umweltpolitischen Handelns ist derjenigen nach den Bedingungen staatlicher Innovationsförderung nahe verwandt. Ebenso wie die empirische Innovationsforschung betont die moderne Policy-Forschung hier die Komplexität und Interaktionsdynamik der Erfolgs- und Innovationsbedingungen von Politik. Nach unseren eigenen wie auch anderen Untersuchungen ergibt sich recht eindeutig, daß erfolgreiche Umweltpolitik nicht nur aus gezieltem (staatlichem und nichtstaatlichem) Handeln erwächst, sondern auch aus der dynamischen Interaktion komplexer Handlungsbedingungen (3). Zentrale Aspekte sind hier der institutionelle Kontext, die Akteurskonstellation, das Politiklernen (policy learning) in Kommunikationsnetzwerken und Verhandlungssystemen, der Politikstil, die situative Seite von Politik, aber auch die Struktur des zu lösenden Problems (4).
Die auf Erfolgsbedingungen von Umweltpolitik konzentrierte empirische Policy-Forschung steht damit im Gegensatz zu herkömmlichen linearen Top-down-Vorstellungen von politischer Steuerung (oder gar „Feinsteuerung"), wie sie in der bisherigen Instrumentendebatte der Umweltpolitik verbreitet sind. Für Innovationświrkungen staatlicher Politik gilt dies erst recht. Staatliche Innovationsförderung ist im Lichte der neueren Policy-Forschung als Feinsteuerung nicht vorstellbar.

Autoren wie Luhmann und Willke hatten mechanistische Steuerungsvorstellungen frühzeitig kritisiert. Luhmann verweist in seiner Abhandlung zur „ökologischen Kommunikation“ (5) auf die Schwierigkeit von politischer Steuerung, die „Resonanzbedingungen“ der Politikadressaten zu treffen und ihren Eigenlogiken, Codes und Programmen gerecht zu werden. Renate Mayntz verwies schon 1983 darauf, daß der Erfolg einer Regulation, abgesehen vom Problem selbst, vom formulierten „Programm“ und der Interaktion zwischen Regulierenden und Regulierten (dem
„Interventionsfeld“) abhänge und mit dem Zielkonsens der Akteure die Bedeutung des Instrumentariums sinke (6). ,Kooperativer Staat" und „dezentrale Kontextsteuerung“ waren dann einige Ersatzformeln. Die Dichotomie von Steuerungsinstanz und Steuerungsobjekt in der herkömmlichen „Gesetzgeberperspektive“ (7) wurde in der Policy-Analyse inzwischen weitgehend aufgegeben. Aus der Forschung zu Erfolgsbedingungen von Umweltpolitik wie aus der empirischen Innovationsforschung ergibt sich als Forschungsthema: Wie lassen sich umweltpolitische Ziele auf dem Wege einer staatlichen Innovationsstrategie realisieren, mit welchen staatlichen Mitteln läßt sich das Tempo des umweltentlastenden technischen Fortschritts signifikant steigern? (vgl. Abbildung) Wie kann eine umweltorientierte Innovationspolitik insbesondere der dynamischen Komplexität der Einflußfaktoren von technischem Wandel gerecht werden?

Die hier formulierte Methodenfrage staatlicher Steuerung ist zugleich eine Methodenfrage sozialwissenschaftlicher Analyse: Die Instrumentenwahl als solche entscheidet über das Ergebnis offenbar weniger als bisher angenommen. Und es ist methodisch in aller Regel kaum möglich, den Beitrag eines einzelnen Instruments von anderen Einflußfaktoren (bzw. Einflußebenen) angemessen zu trennen.

\section{Zwei konkurrierende Ansö̈ze}

Auf dem Markt der Wirkungsanalyse staatlichen Handelns konkurrieren vor allem zwei Erklärungsansätze: a) der modelltheoretische Ansatz der Ökonomie und b) der Ansatz der Evaluationsforschung bzw. der empirischen PolicyAnalyse, der dem der empirischen Innovations-

Umweltpolitische Zielbildung und Instrumentierung einer innovationsorientierten Zielgruppenstrategie

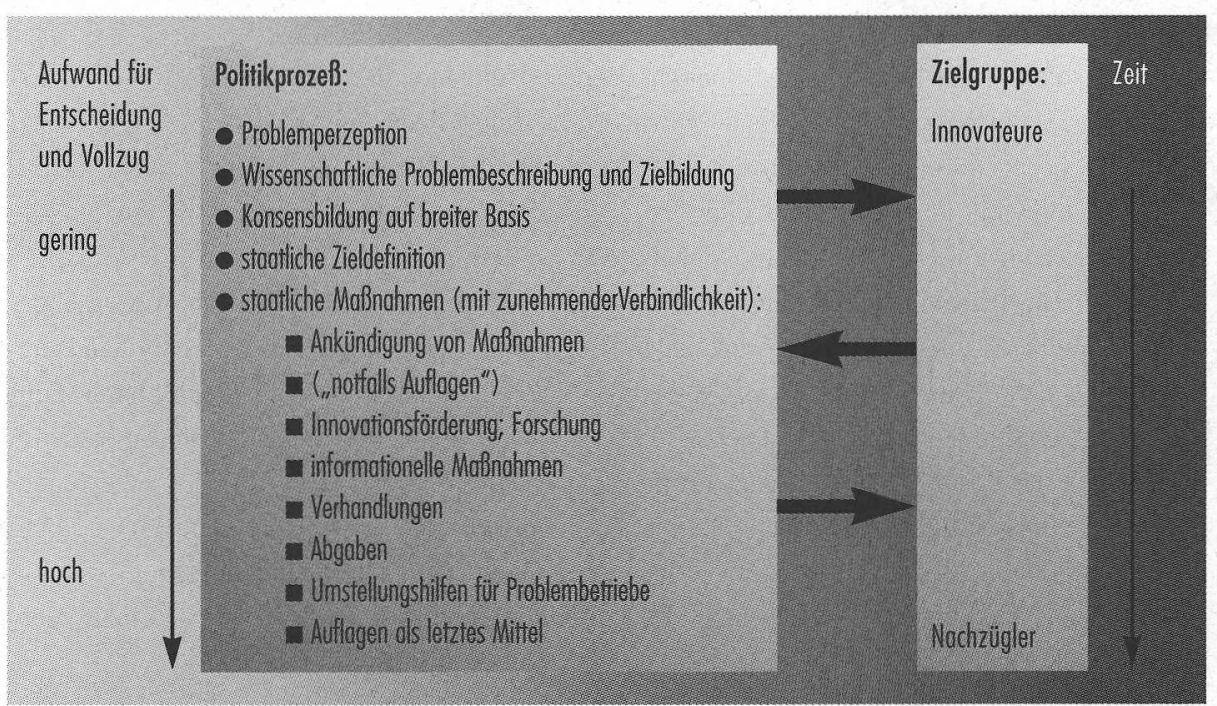


forschung sehr verwandt ist. Der erstgenannte Ansatz untersucht die Wirkungen politischer Maßnahmen in aller Regel ex ante und top down, d. h. aus der Sicht des staatlichen Maßnahmenträgers (8). Zumeist ist das kein strikt empirisches Vorgehen. Bei kritischer Betrachtung könnte gesagt werden: Der Ansatz arbeitet mit zu we- nigen Variablen und bemüht zuviel Plausibilität. Die gewählten Annahmen sind dabei offen für allerlei Interessenseinflüsse: Daher auch die Breite der Kontroverse um entsprechende Forschungen.

Die Wirkungsforschung der Policy-Analyse beginnt häufig ebenfalls ex ante und top down. Aber im Kern evaluiert sie empirisch eingetretene Effekte ex post. Lange war sie reine Begleitforschung für politische Maßnahmen, insoweit auf staatliches Handeln bezogen. Neuere Ansätze der Umweltpolitikforschung stellen aber den bottomup-Ansatz ganz in den Vordergrund und forschen mitunter regelrecht von den Wirkungen hin $\mathrm{zu}$ den Ursachen. Damit ist die Evaluation methodisch offen für jede Art möglicher Einflußfaktoren, seien sie staatliches Handeln oder andere Determinanten. Ein solcher Zugang überschreitet zwangsläufig enge Disziplingrenzen. Als Problem ergibt sich hier der Umgang mit Überkomplexität, während der ökonomische Ansatz diese meist übermäßig vereinfacht.

Die Policy-Analyse, die ex post Erfolge oder Mißerfolge von Politik zu erklären sucht, ist dem Ansatz der empirischen Innovationsforschung nicht nur ähnlich, sondern häufig auf diesen bezogen. Eine Kombination beider Ansätze erscheint ebenso unkompliziert wie naheliegend: Der Policy-Ansatz wäre in diesem Fall politikzentriert, der der Innovationsforschung unternehmenszentriert. Die Bedingungen und Eigenlogiken beider Handlungsfelder wären dann systematisch aufeinander zu beziehen. Den Innovationsbedingungen von Unternehmen wären die Erfolgsbedingungen von Umweltpolitik zuzuordnen.

\section{- Methodische Probleme des Top-down-Ansatzes}

Das entscheidende Problem der ökonomischen ex-ante-top-down-Modellierung ist es, Prognosen über die Wirkung isoliert betrachteter staatlicher Maßnahmen zu machen. Was das bedeutet, soll an einem Extrembeispiel aus unseren Untersuchungen verdeutlicht werden:

Die japanische Industrie hatte von 1974 bis Ende der achtziger Jahre einen progressiven Stromtarif, der zudem im OECD-Vergleich lange weit an der Spitze lag. Die Gründe - teure Energieimporte und Kapazitätsengpässe der Stromwirtschaft müssen hier nicht interessieren. Was hätten uns ökonomische Modellanalysen ex ante im Jahre 1973 hierzu mitgeteilt? Mit hoher Wahrscheinlichkeit wären schwere kosteninduzierte Wettbewerbsprobleme für die stromintensiven Industrien prognostiziert worden (Chemie, Metallurgie etc.). Tatsächlich lag das Wachstum der Chemieindustrie von 1971 bis 1987 noch über dem industriellen Durchschnitt. Der Stromverbrauch aber nahm kaum zu. Dies ist im internationalen Vergleich eine völlig atypische Entwicklung (9), die nur durch massive Innovationen erklärt werden kann. Und diese können hier vorrangig auf die, einer staatlichen Abgabenlösung gleichkommende, Struktur der Stromtarife zurückgeführt werden. Dabei darf allerdings von der flankierenden staatlichen Technologiepolitik und einer engen Konzertierung von Staat und Industrie nicht abgesehen werden.

Wie läßt sich dies sinnvoll in Modellrechnungen simulieren? Eine nachträgliche Evaluation, die den tatsächlichen Einflußfaktoren Rechnung trägt, hat es hier leichter. Freilich: sie kommt nachträglich. Daher liegt eine entsprechende Kopplung beider Ansätze nahe.

Während im angefiuhrten Fall die Innovationswirkung vermutlich wirklich auf einen dominanten Lenkungsfaktor zurückgefuihrt werden kann, ist dies bei der Wirkungsanalyse umweltpolitischer Instrumente in der Regel methodisch ungleich schwieriger. Das liegt zunächst einmal daran, daß zumeist ein policy-mix vorliegt. Wir haben in unseren international vergleichenden Untersuchungen kaum einen Fall gefunden, wo nur eine einzelne Maßnahme die erwünschte Wirkung erzielte. Das Methodenproblem lautet hier: wie lassen sich einzelne Instrumente in einem Mix kausalanalytisch isolieren? Aber das ist nicht das einzige zu lösende Methodenproblem. Es treten weitere Konstellationen auf, die sich in den empirischen Untersuchungen der Forschungsstelle für Umweltpolitik gezeigt haben (10):

- Vermeintlich eindeutig auf einzelne Instrumente zurückführende Umweltwirkungen basieren bei näheren Hinsehen auf einem breiten Instrumentenspektrum.

- Identische Erfolge wurden verschiedenen Ländern mit sehr unterschiedlichen Mitteln erzielt (z.B. Gewässerschutz in Holland (Abgaben) und Schweden (Subventionen)).
- Oft ist es nicht primär die konkrete Instrumentenwahl, die zählt, sondern der Signaleffekt der Maßnahme als solcher.

- Grund zur methodischen Vorsicht geben insbesondere jene Fälle von Umweltverbesserungen, die nicht auf direkte staatliche Maßnahmen $\mathrm{zu}$ rückzuführen sind, gleichwohl aber mit informellem Verwaltungshandeln verbunden sein können. Unter dem Innovationsaspekt führen die letzten Aspekte zu einem entscheidenden Punkt: Substitutionen und innovative Anpassungsprozesse der Industrie erfolgen oft, bevor es zu einer förmlichen Maßnahme des Staates kommt (11). Aus den Ergebnissen der empirischen Untersuchungen lassen sich dabei neue Formen von Regulierungsmustern entwickeln, die die Anforderungen einer innovativen Umweltpolitik erfiillen.

\section{Top-down und Bottom-up: mögliche Vermittlungen}

In der Instrumentenfrage ist also ein Paradigmenwechsel fällig und bereits auch im Gange, der zugleich auf einen umfassenden Forschungsbedarf über neue Formen staatlicher Steuerung verweist. Aus der Sicht der empirischen Evaluation von umweltpolitischem Handlungserfolg ergibt sich heute ein sehr differenziertes Bild des staatlichen Instrumentariums. Es ist dies ein anderes Bild, als es Modellanalysen einzelner Instrumente ergeben. Für die Wirkungsanalyse von Umweltsteuern haben dies unlängst auch die OECD und die Europäische Umweltagentur betont (12). Beispielsweise können niedrigere Abgaben in Kombination mit anderen Instrumenten höhere Lenkungswirkungen erzielen als Modellrechnungen mit vorgegebenen Elastizitätsannahmen dies ergeben. Selbst niedrige Abgaben können bereits die Signalwirkung entfalten, die für Innovateure relevant sind. Das Verdienst der herkömmlichen Instrumentendebatte ist es allerdings, das Bewußtsein über mögliche Handlungsoptionen staatlicher Umweltinstitutionen verbessert zu haben.

Eine Vermittlung dieses Top-down-Ansatzes mit den Erkenntnissen einer Evaluationsforschung, die vom empirischen Erfolgsfall ausgeht, erscheint gerade dann sinnvoll, wenn die Innovationswirkungen staatlichen Handelns das Thema sind. Dieser Vermittlung widmet sich die FFU in ihren aktuellen Forschungsarbeiten. Dies ist zugleich ein gutes Beispiel für einen Forschungsansatz, der dem Anspruch transdisziplinärer Forschung (Problembezug, Überwindung disziplinärer Grenzen) gerecht wird. 


\section{Anmerkungen}

(1) Vgl. Héritier, A. (Hrsg.): Policy-Analyse. Kritiik und Neu-orientierung, Politische Vierteliahresschrift, Sonderheft 24, Opladen 1993;

Prittwitz, V. v.: Politikanalyse, Opladen 1994;

R. Rist: Policy Evaluation. Linking Theory to Practice, Cheltenham 1994;

Rossi, P. H./H. Freeman, E.: Evaluation. A Systematic Approach, Newbury Park, London, New Delhi 1993 (5. Aufl.);

Fischer, F.: Evaluating Public Policy, Chicago 1995.

(2) Vgl. Jänicke, M. (Hrsg.): Umweltpolitik der Industrieländer. Berlin 1996.

(3) Vgl. Jänicke, M., Weidner, H. (eds.): Successful Environmental Policy. A Critical Evaluation of 24 Cases.

Berlin 1995;

Conrad, J.: Successful Environmental Management in European Companies, FFU-report 96-3, Berlin 1996.

(4) Vgl. Jänicke, M. (Hrsg.) a.a.0. 1996; und Mayntz, R.: Zur Einleitung: Probleme der Theoriebildung in der Implementationsforschung, in: Dies. (Hrsg.): Implementation politischer Programme, Opladen 1983.

(5) Luhmann, N.: Ökologische Kommunikation, Opladen 1986.

(6) Mayntz, R. a.a.0. 1983.

(7) Willke, H.: Entzauberung des Staates Überlegungen zu einer sozietalen Steuerungstheorie, Königstein/Ts 1983.

(8) Sabatier, P. A.: Top-down and Bottom-up Approaches to Implementation Research, in: Hill, M. (Ed.): The Policy Process: A Reader, London 1993.

(9) M.Jänicke, H.Mönch, M.Binder, et al.: Umweltentlastung durch industriellen Strukturwandel? Berlin 1993.

(10) Vgl. hierzu Jänicke, M.: Umweltinnovationen aus

Sicht der Policy-Analyse: vom instrumentellen zum strategi-

schen Ansatz der Umweltpolitik. FFU-rep. 97-3,

Berlin 1997.

(11) Vgl. zu entsprechenden Studien am Beispiel der

Reduktion von Gefahrstoffen: Jacob, K., Jänicke, M.: Ökologische Innovationen in der chemischen Industrie: Umweltentlastung ohne Staat? Eine Untersuchung und Kommentierung zu 182 Gefahrenstoffen. FFU-rep 97-4,

Berlin 1997.

(12) OECD: Environmental Taxes and Green Tax Reform, Paris 1997;

European Environment Agency (EEA): Environmental Taxes. Implementation and Environmental Effectiveness, Copenhagen 1996.

\section{Der Autor}

Prof. Dr. Martin Jänicke ist Prolessor für Politikwissenschaft und Leiter der Forschungsstelle fïr Umweltpolitik an der FU Berlin (FFU)

Kontakı: Martin Jänicke, FFU

Schwendener Straße 53, 14195 Berlin

Tel. $++49 /(0) 30 / 8385098$

Eine transdisziplinäre Gestaltungsaufgabe der Politikberatung

\title{
Institutionen für eine nachhaltige Gesellschaft
}

\author{
Sozialwissenschaftliche Schlïsselkonzepte wie dasjenige der "Institution" \\ eröffnen ebenfalls den Zugang zu einer transdisziplinären Wissensgenerierung. \\ Dabei ist die instifutionelle Perspektive von hoher theoretischer und prakti- \\ scher Relevanz in der akfuellen politischen Debatte über die Umsetzung des \\ Konzeptes der Nachhaltigkeit.
}

Von U. Schneidewind, J. Minsch, T. Schulz, J. Wüst, H. P. Meister, P. Feindt, J. Tscheulin

$\mathrm{D}$ ie Diskussion über eine Politik der Nachhaltigkeit läuft heute in vielen Feldern in eine ,Ziel-Instrumenten-Falle": Weithin besteht die Vorstellung, daß in einem ersten Schritt konkrete ökonomische, ökologische und soziale Nachhaltigkeitsziele zu definieren und sie in einem zweiten Schritt mit konkreten Instrumenten umzusetzen seien. Diese auf den ersten Blick plausible Vorstellung abstrahiert jedoch völlig von den konkreten Formen der Entscheidungsfindung in der Gesellschaft sowie den Motivationen der beteiligten Akteure. Die Ergebnisse sind ernüchternd: Die politische Diskussion über Nachhaltigkeit verfängt sich in blockierten Zieldiskussionen und gleichzeitig bleiben Gestaltungspotentiale auf zahlreichen gesellschaftlichen Ebenen ungenutzt. Eine transdisziplinär angelegte Institutionenanalyse kann hier wichtige Impulse für eine nachhaltigkeitsorientierte Politikberatung (1) geben.

\section{- Renaissance der Institutionenperspektive}

Die Auseinandersetzung mit Institutionen spielt in der Politologie, der Soziologie und den Wirtschaftswissenschafen von je her eine wichtige Rolle - in der Regel bezogen auf die spezifischen Fragestellungen der Disziplin. Insbesondere die Volkswirtschaftslehre hat mit der Neuen Institutionenökonomik eine Antwort auf zahlreiche Erklärungsdefizite enger neoklassischer Ansätze entwickelt. Die Etablierung des institutionellen Zweigs der VWL fand nicht zuletzt ihren Ausdruck in den Nobelpreisverleihungen an Ronald Coase und Douglas C. North. Die Endogenisierung von
Institutionen ist dabei eines der zentralen Anliegen der Neuen Instititutionenökonomik (2). Bezogen auf Ihre Anwendungsgebiete ist die Neue Institutionenökonomik interdisziplinär angelegt: Sie beschränkt ihre Analyse nicht nur auf marktliche Strukturen, sondern läßt sich ebenso auf politische Strukturen anwenden. Sie kann als Ausdruck eines ,NeoInstitutionalismus" auch in den anderen Gesellschaftswissenschaften betrachtet werden. Dieser Neo-Institutionalismus repräsentiert eine Art ,Wiederentdeckung" der institutionellen Ebene sozialen Handelns und versucht unter Rückgriff auf Disziplinen wie Politologie, Soziologie, Volkswirtschaftslehre, Rechtswissenschaft, Anthropologie und Philosophie einen interdisziplinär begründeten Mittelweg zwischen akteurszentrierten und funktionalistischen Erklärungsmodellen gesellschaftlicher Prozesse zu entwickeln.

Doch ist die Institutionenanalyse heute nicht nur theoretisch wieder ,en vogue“. Die theoretische öffnung hin $\mathrm{zu}$ Institutionen wird begleitet von einer intensiven Diskussion über Institutionen in der heutigen Gesellschaft. Dies bezieht sich nicht nur - wie in der Einleitung angedeutet - auf die Ergänzung der Zieldiskussion in der Nachhaltigkeitsdebatte um die institutionellen Voraussetzungen einer nachhaltigen Gesellschaft, sondern immer mehr auf die Frage, wie Politik heute insgesamt zu organisieren ist. Zunehmende staatliche Steuerungsdefizite oder Politikverdrossenheit auf der einen Seite, gleichzeitig neue selbstorganisierte Formen der gesellschaftlichen Problemlösung (Branchenselbstverpflichtungen, lokales Engagement etc.) auf der anderen Seite verlangen nach neuen institutionellen Gestaltungsoptionen. 
(c) 20I0 Authors; licensee IÖW and oekom verlag. This is an article distributed under the terms of the Creative Commons Attribution Non-Commercial No Derivates License (http://creativecommons.org/licenses/by-nc-nd/3.o/), which permits unrestricted use, distribution, and reproduction in any medium, provided the original work is properly cited. 